\title{
Editorial
}

\section{Scaling, Self-Similarity, and Systems of Fractional Order}

\author{
Carlo Cattani, ${ }^{1}$ Martin Ehler, ${ }^{2}$ Ming Li, ${ }^{3}$ Zhiwu Liao, ${ }^{4}$ and Mohammadreza Hooshmandasl ${ }^{5}$ \\ ${ }^{1}$ Department of Mathematics, University of Salerno, Via Giovanni Paolo II, 84084 Fisciano, Italy \\ ${ }^{2}$ Faculty of Mathematics, University of Vienna, Oskar-Morgenstern-Platz 1, 1090 Vienna, Austria \\ ${ }^{3}$ Institute of Systems Science, East China Normal University, Shanghai 200241, China \\ ${ }^{4}$ Visual Computing and Virtual Reality Key Laboratory of Sichuan Province, Sichuan Normal University, Chengdu 610101, China \\ ${ }^{5}$ Faculty of Mathematics, Yazd University, Yazd, Iran \\ Correspondence should be addressed to Carlo Cattani; ccattani@unisa.it
}

Received 3 August 2014; Accepted 3 August 2014; Published 31 August 2014

Copyright (c) 2014 Carlo Cattani et al. This is an open access article distributed under the Creative Commons Attribution License, which permits unrestricted use, distribution, and reproduction in any medium, provided the original work is properly cited.

Scaling (power-type) laws and self-similarity reveal some featuring properties of physical-chemical objects and can be easily noticed in nature. Moreover, also some mathematical abstract objects, such as nondifferentiable functions and fractals, enjoy scaling and self-similarity. Experimental data often show some characteristic power law and self-similarity. A self-similar (scaling) object repeats itself at different scales in space or time. The property of self-similarity gives us a better opportunity to study phenomena from all analytical and computational aspects.

Scale dependence and multiscale analysis are peculiar properties of some families of special functions and can be observed in nature. A continuous scale transformation from one scale to another implies a generalization and suitable extension of differential operator, as it happens with fractional derivatives.

Dynamical processes and systems of fractional order attract researchers from many areas of sciences and technologies, ranging from mathematics and physics to computer science. From analytical point of view, these kinds of problems often lead us to deal with the concepts of scales, fractals, and fractional operators. For instance, medical images nowadays play an essential role in detection and diagnosis of numerous diseases and a suitable scale-depending interpretation of the images is a fundamental aspect of the clinical investigation. Nonlinear analysis of data, collected by modern devices, offers still unsolved analytical problems related to not only complex physics and abstract mathematical theories but also nonlinear science.
The focus of this special issue is on both the abstract mathematical models on scaling and self-similarity and the applied computations on those dynamical processes and systems of fractional order towards the applications in all aspects of theoretical and practical study in analysis.

Scaling and self-similarity characterize several mathematical topics:

(1) self-similar analytical problems: scale-depending theoretical and applied analytical problems;

(2) fractals, nondifferentiable functions: theoretical and applied analytical problems of fractal type;

(3) $1 / f$ process, fractional Brownian motion, fractional Gaussian noise, self-similar processes, long memory processes, heavy-tailed random processes, and power law systems;

(4) fractional differential/integral equations, fractional operators: systems of fractional order;

(5) complex systems, nonlinear processing;

(6) wavelets;

(7) scaling and self-similarity in applications by focusing on theoretical and analytical aspects arising, for example, in nonlinear analysis of data, image analysis, data science, and system science.

This special issue contains 17 papers.

In the category of scale-depending problems, fractals and, self-similarity there are many papers devoted to interesting problem. 
H. Zhai proposes some discussion on certain modular equations about infinite products of Ramanujan. The paper of J. Leng and T. Huang deals with the "Construction of fusion frame systems in finite dimensional Hilbert spaces." The fractional order, in particular "On the $(p, q)$ th relative order oriented growth properties of entire functions," has been investigated in the paper of L. M. S. Ruiz et al. The convergence analysis of the numerical approximation of second kind integral equations by hybrid Bernstein blockpulse functions has been proposed by M. Alipour et al. The paper of C. Pang et al deals with the complex problem of "Applying data clustering feature to speed up ant colony optimization."

In their paper, $\mathrm{S}$. Hu, and P. Liang propose a smart model to detect and locate singularities by using the theory analysis of left-handed Grünwald-Letnikov formula with $0<\alpha<1$.

J. Yang et al. investigate the "Nonrigid registration of monomodal MRI using linear viscoelastic model." The "Linear total variation approximate regularized nuclear norm optimization for matrix completion" has been discussed in the paper of X. Han et al. The " $\varepsilon$-coverings of Hölder-Zygmund type spaces on data defined manifolds" are the main topics of the paper of M. Ehler and F. Filbir. In the paper of L. Dong et al., "Nonlinear methodologies for identifying seismic event and nuclear explosion using random forest, support vector machine, and naive bayes classification" are studied.

Several papers are dealing more specifically with fractional calculus-systems of fractional order.

Transforms within the theory of local fractional calculus are considered, respectively, in the paper of X. Yang et al. by focusing on the continuous wavelet transform and the paper of $\mathrm{K}$. Liu et al. which is dealing with the "Local fractional $Z$ transforms with applications to signals on cantor sets."

Approximate solution of fractional differential equations is considered in the paper "Picard successive approximation method for solving differential equations arising in fractal heat transfer with local fractional derivative" proposed by A. Yang et al. and in the paper of J. Duan et al. dealing with the "Similarity solution for fractional diffusion equation."

A new definition of fractional derivatives based on truncated left-handed Grünwald-Letnikov formula with $0<\alpha<$ 1 and median correction has been investigated by Z. Liao.

Z. Deng and X. Yang propose a "Discretized Tikhonov regularization method for a fractional backward heat conduction problem."

Mathematical models arising in the fractal forest gap via local fractional calculus are studied in the paper of C. Long et al.

Of the 36 submissions, 17 papers are accepted in this special issue (with the acceptance rate being 47.2\%). All papers are dealing with current problems in the topics; however, they are not an exhaustive representation of the area of fractional order systems where the concepts of scale, self-similarity, and fractional order interact. In all papers, the authors have focused on the main aspects of the theory and although they have proposed some solutions and models, most problems remain open, thus giving the opportunity to readers for further research and discussions in this field.

\section{Acknowledgment}

Thanks to the excellent authors' contributions, all of the key aspects raised have been addressed. The authors would also thank the reviewers in helping improvement of the papers and the publisher for continuous professional assistance. One editor (Ming Li) acknowledges the supports in part by the National Natural Science Foundation of China under the Project Grants nos. 61272402, 61070214, and 60873264.

Carlo Cattani Martin Ehler Ming $\mathrm{Li}$ Zhiwu Liao Mohammadreza Hooshmandasl 


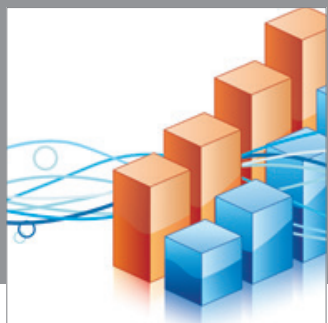

Advances in

Operations Research

mansans

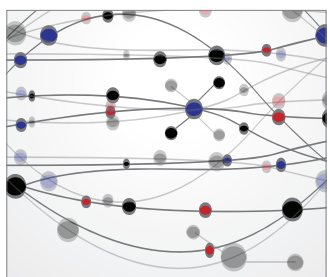

The Scientific World Journal
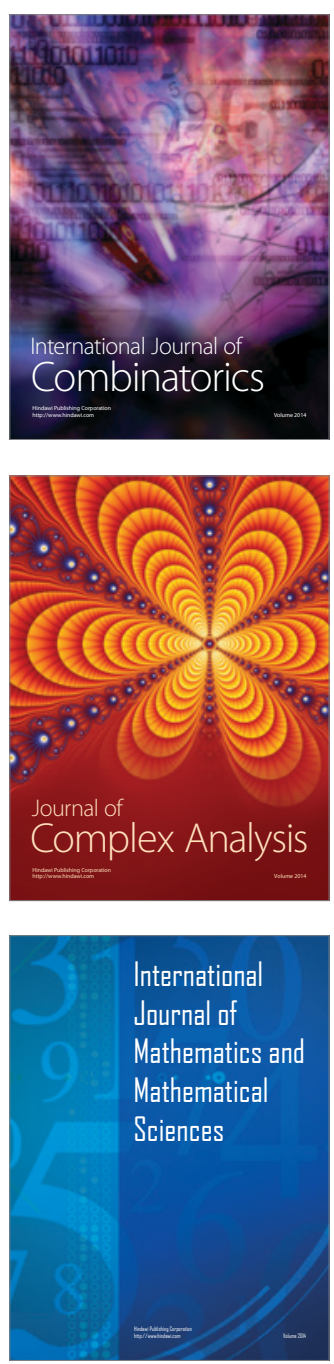
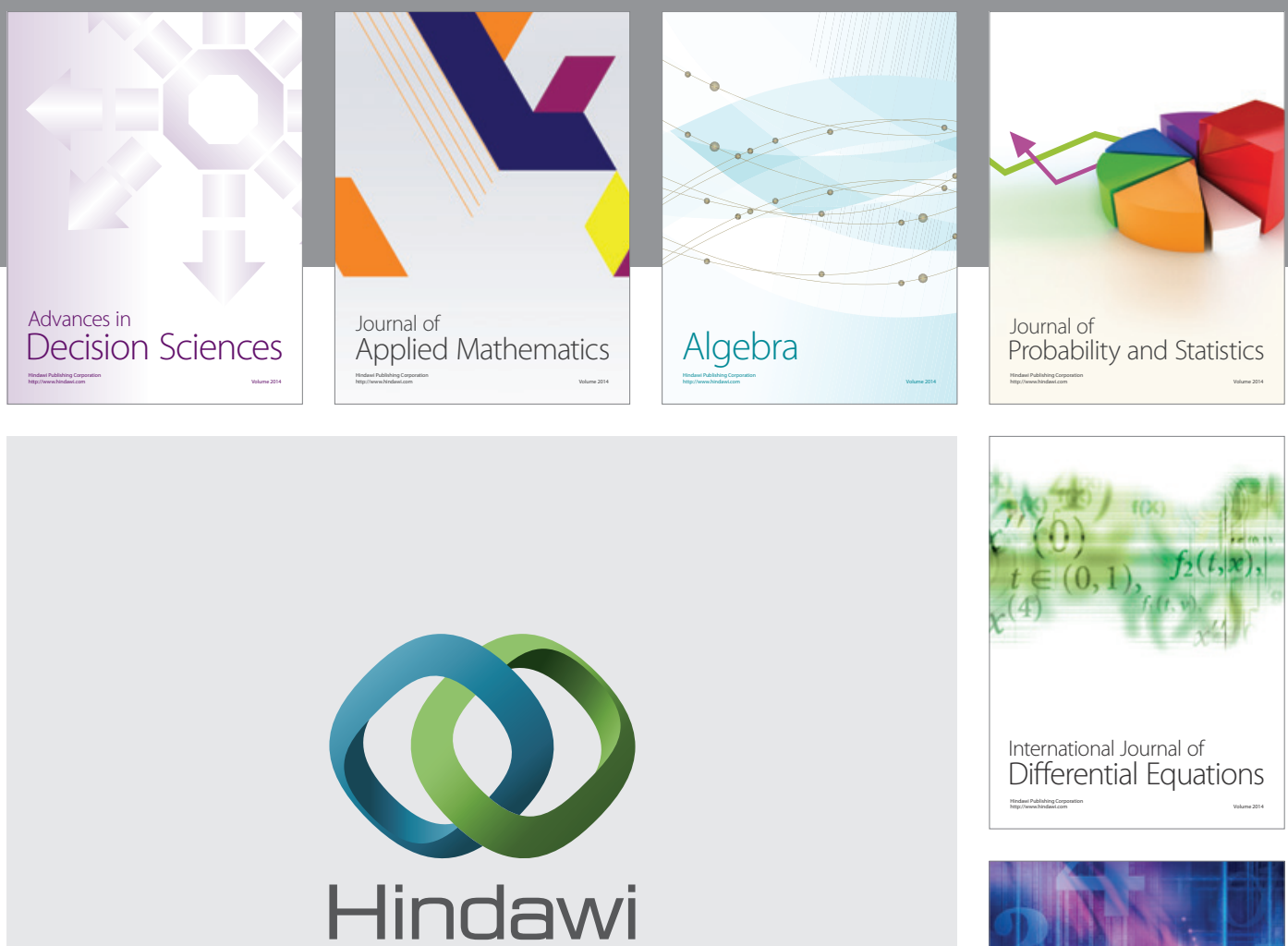

Submit your manuscripts at http://www.hindawi.com
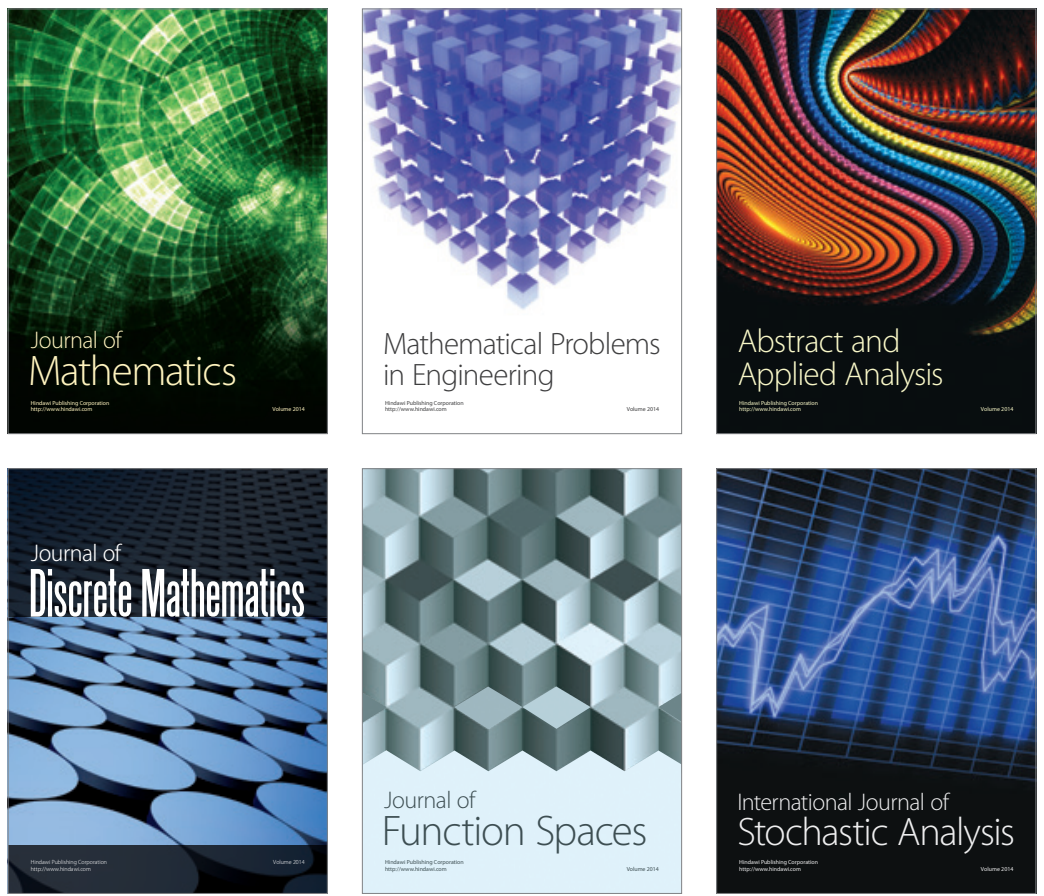

Journal of

Function Spaces

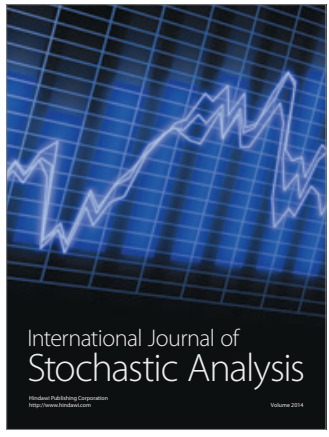

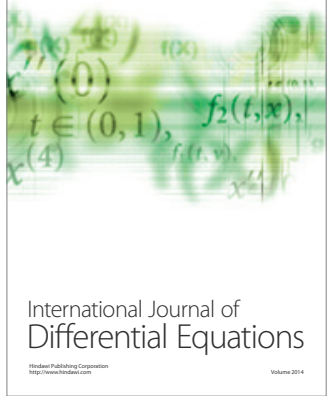
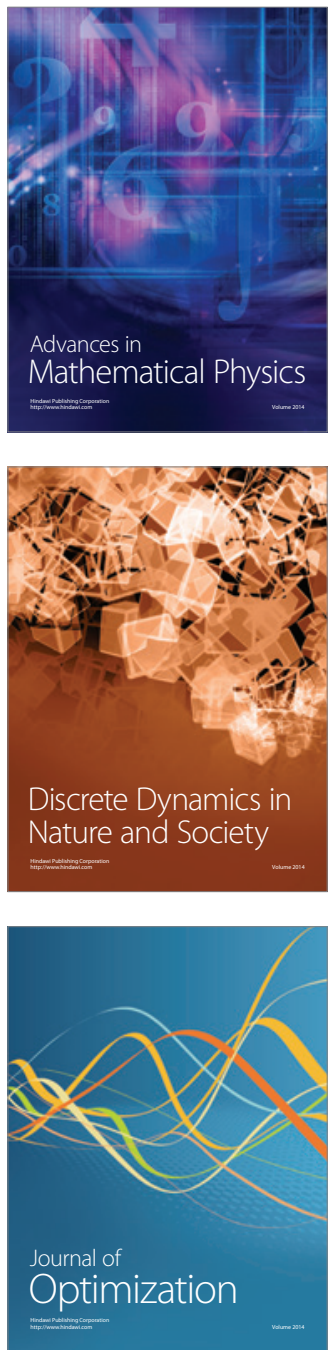